\title{
Inhibitory effect of plant metabolites of Nigella sativa on conditionally pathogenic microflora of productive animals
}

\author{
Anna Krivonogova ${ }^{2}$, Albina Isaeva ${ }^{1,2, *}$, Antonina Poryvaeva ${ }^{2}$, Anastasia Chentsova $^{1}$, and \\ Pavel Sharavyev ${ }^{1}$ \\ ${ }^{1}$ Ural State Agrarian University, Department of Infectious and Non-infectious Pathology, 620075 \\ Yekaterinburg, Russia. \\ ${ }^{2}$ Federal State Budgetary Scientific Institution «Ural Federal Agrarian Scientific Research Centre, \\ Ural Branch of Russian Academy of Sciences», 620142 Yekaterinburg, Russia
}

\begin{abstract}
Objective: The effectiveness of a phytobiotic based on active metabolites of Nigella sativa L. against typical microorganisms of opportunistic microbiocenosis of the mucous membranes of highly productive cows was studied. Initially, the inhibitory activity of the phytobiotic was studied on cultures of wild multi-antibiotic-resistant isolates of $\mathrm{P}$. aeruginosa and $\mathrm{S}$. aureus isolated on a commercial dairy farm. It was found that the phytobiotic had the ability to inhibit the growth of isolates on the nutrient medium, but the severity of the inhibitory effect varied notably. At the second stage, an experiment was conducted with the local application of phytobiotics on cows that had inflammatory complications of the postpartum period. The results of the experiment showed a pronounced inhibitory effect of the phytobiotic on S. aureus, Str. uberis, P. aeruginosa, E. cloacae, C. albicans, and P. mirabilis. There was an involution of inflammatory symptoms and normalization of the clinical and microbiological state of the mucous membrane after the use of a phytobiotic preparation.
\end{abstract}

\section{Introduction}

Microbiocenosis of the mucous membranes of the reproductive system in cows is one of the key factors that play a role in the development of postpartum inflammatory complications. The predominance of pathogenic microorganisms in the microbiocenosis structure significantly increases the risk of endometritis and slows down the rate of regeneration of the birth canal tissues. In case of acquired immunodeficiency in combination with physiological immunosuppression during pregnancy, any opportunistic microflora can cause an inflammatory process in the tissues of the mucous membrane of the vagina and uterus, which, in turn, has a negative effect on the entire body $[1,2]$. Currently, antibiotics are traditionally used to correct dysbiosis and treat pathologies caused by opportunistic microflora. Nevertheless, the rapid spread of antimicrobial resistance is

\footnotetext{
* Corresponding author: isaeva.05@bk.ru
} 
increasingly making antibiotics ineffective. An alternative treatment option for dysbiosis and certain infectious conditions is phytobiotics, which are used in combination with antibiotics, less often - as an independent therapeutic agent [3-7]. The mechanisms of action of the main plant metabolites with antimicrobial activity - phytoncides, terpenoids, phenolic lipids, isothiocyanates, tannins, organic acids, and flavonoids - are known and studied $[7,8]$. The bactericidal effect of most phytobiotics is mediated through the effects of membrane toxicity, inhibition of membrane transport proteins, changes in the permeability and destruction of lipophilic components of cell membranes [4,6,7]. At the same time, active substances of plant nature exhibit bacteriostatic, bactericidal properties both against gram-positive and gram-negative microorganisms, including S. aureus, P. aeruginosa, E. coli, E. feacalis, C. albicans, which are facultative pathogens inhabiting the mucous membranes of animals [6-9]. The study of the local antimicrobial activity of phytobiotics and the development of methods for their external use will allow to supplement or replace traditional antibiotic therapy schemes for the correction of dysbiosis of the mucous membranes of productive animals, which is certainly very important in the context of growing antimicrobial resistance.

\section{Materials and Methods}

The study of the inhibitory effect of an external phytobiotic on typical microorganisms of opportunistic microbiocenosis of the mucous membranes of highly productive cows was carried out. The preparation was a water-oil emulsion for external use, which includes: distilled water $(74 \%$ vol.), cold-pressed Nigella sativa L oil $(25 \%$ vol.), essential oil of Nigella sativa L. seeds, obtained by pressing and steam distillation $(1.0 \%)$. The basis for the preparation was chosen because of the high content of thymoquinone and thymohydroquinone in N. sativa oils, which are known for their activity against S. aureus, P. aeruginosa, E. coli and C. albicans $[8,9,10]$. The mass fraction of active metabolites in the essential oil component was as follows: thymoquinone - $32.8 \%$, thymohydroquinone $2.7 \%$, longifolene $-1.7 \%$. Essential oils were produced on the basis of FSBIS Agricultural Research Institute of the Crimea.

At the first stage, the inhibitory activity of the preparation was studied on cultures of multi-resistant microorganisms Staphylococcus aureus and Pseudomonas aeruginosa. Isolates of microorganisms were previously isolated from swabs from the mucous membranes of cows on commercial dairy farms, and their sensitivity to antibiotics was analyzed by the disc diffusion method according to standard microbiological methods [11]. To test the phytobiotic preparation, 16 isolates were selected that had the following antibiotic sensitivity profiles: P. aeruginosa - simultaneous resistance to meropenem, enrofloxacin and tobramycin; S. aureus - simultaneous resistance to benzylpenicillin, amoxicillin, meropenem, tetracycline and enrofloxacin. The analysis of the phytobiotic inhibitory effect was performed by volume displacement method on Muller-Hinton agar. The inhibition zone of microorganisms was analyzed. The following criteria were taken into account: inhibition zone of less than $6 \mathrm{~mm}$ - the isolate is not sensitive to the preparation, 7-16 $\mathrm{mm}$ - moderate sensitivity, more than $16 \mathrm{~mm}$ - the isolate is sensitive.

At the second stage, we studied the local antimicrobial effect of a phytobiotic based on the metabolites of Nigella sativa in vivo, on the basis of the commercial dairy farm. For the experiment, 10 cows were selected in the postpartum period, which had clinical signs of dysbiosis and inflammatory process of the vaginal mucosa. The phytobiotic preparation was applied to the mucous membrane by irrigation in the amount of $20 \mathrm{ml}$ once per 1 head per day for 7 days. Before and after the experiment, swabs were taken from the vaginal mucosa for microbiological analysis. The microorganisms were cultured on dense nutrient 
media, identified by the MALDI-TOF method in the VITEK MS analyzer, and pathogenicity was determined by standard microbiological methods.

Processing of the obtained results was carried out in the program STATISTICA 10, the plan and methodology of the experiment on farm animals were approved by the Ethical Commission of the Ural State Agrarian University.

\section{Results and discussion}

The results of the study of the inhibitory activity of the phytobiotic based on secondary plant metabolites of Nigella sativa L., conducted on cultures of microorganisms, showed that the majority of multi-resistant isolates of S. aureus and P. aeruginosa were sensitive to the preparation to some extent (Figure 1).

\section{Distribution of experimental isolates by sensitivity to phytobiotic based on metabolites of Nigella sativa $(n=16))$}

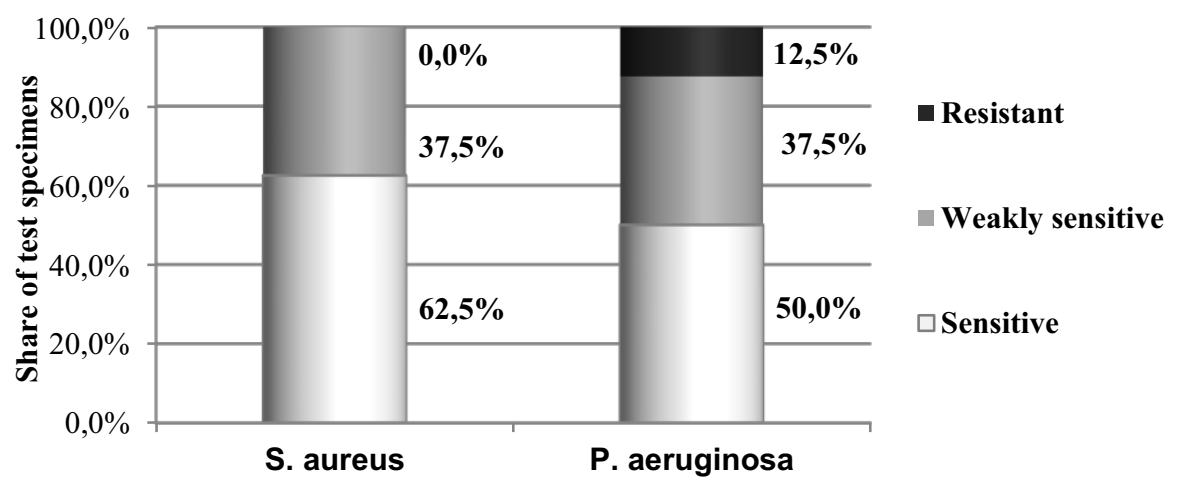

Fig. 1. Cultures of microorganisms

Of the eight $\mathrm{S}$. aureus isolates, more than a half showed good sensitivity. The diameter of the inhibition zone in the phytobiotic-sensitive isolates varied from $18 \mathrm{~mm}$ to $44 \mathrm{~mm}$. In moderately sensitive S. aureus, the inhibition zone was 8,10 , and $11 \mathrm{~mm}$; no stable isolates were found in the experiment. Thus, the variability of the response of $\mathrm{S}$. aureus isolates to the phytobiotic placed in the lunula on the nutrient medium was noted. Presumably, it is associated with the ability of individual $\mathrm{S}$. aureus isolates to maintain viability at different concentrations of the phytobiotic active components in the nutrient medium; Supposably, it causes a difference in the size of the growth inhibition zones and has a positive correlation with the concentration gradient of the phytobiotic at a distance from the well to the periphery.

Multiresistant isolates of $P$. aeruginosa showed a satisfactory average sensitivity to the phytobiotic based on Nigella sativa L. oils, but compared to S. aureus, the result was worse due to the presence of resistant isolates. In general, half of the isolates showed a good reaction to the phytobiotic - the diameter of the growth inhibition zone was $20-31 \mathrm{~mm}$, on average it was about $26 \mathrm{~mm}$. The worst result was about $2 \mathrm{~mm}$. The results of the group with moderate sensitivity were in the range of 7-16 $\mathrm{mm}$.

In the experiment conducted on cows in the postpartum period, a positive effect of local phytobiotic treatments on the microbiocenosis of the vaginal mucosa was established. In the swabs taken before the start of the experiment, in cows in the early postpartum period (1216 days after calving), P. aeruginosa, S. aureus, E. faecalis, E. faecium, P. vulgaris, P. mirabilis, E. coli, H. somni, Str. uberis, Penicillium spp., C. albicans, Bacillus spp. were 
detected in large quantities. In cows 30-33 days after calving, P. aeruginosa, S. aureus, Str. uberis, H. somni, E. faecalis, E. faecium, E. cloacae, E. coli, P. vulgaris, Bacillus spp., C. albicans, Penicillium spp. were mainly detected. Fecal enterococcus was detected in $70 \%$ of the animals in the group, Staphylococcus aureus - in $60 \%$, and escherichia coli - in $90 \%$ (Figure 2).

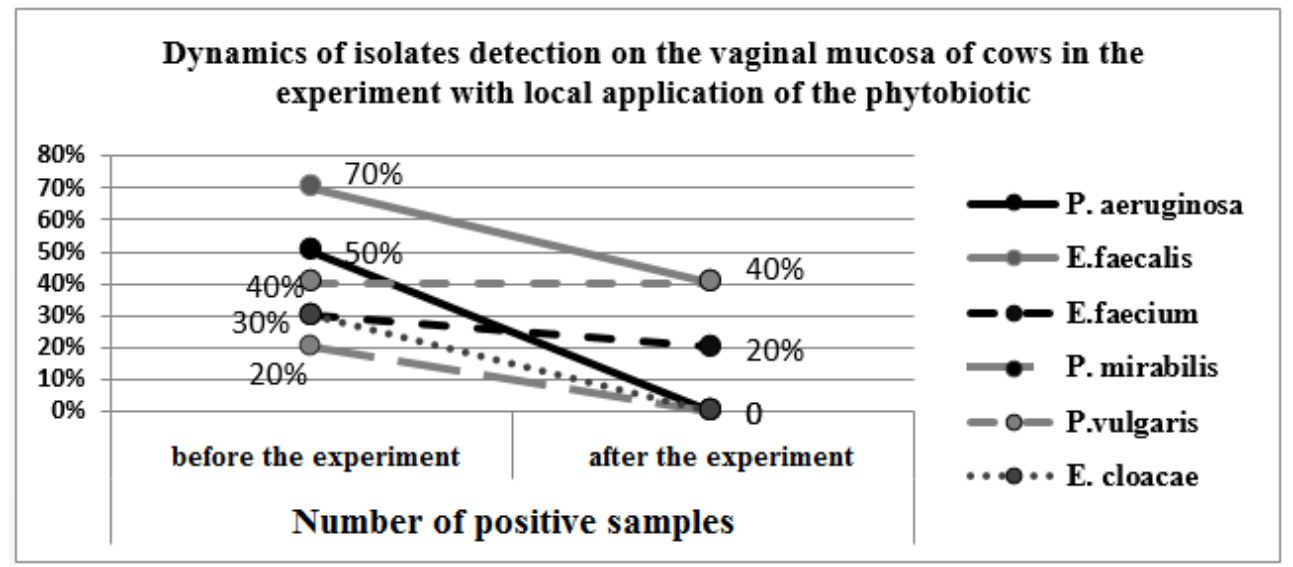

Fig. 2. Dynamics of OM isolates detection on the vaginal mucosa of cows in the experiment with local application of the phytobiotic based on Nigella sativa L.

After a course of phytobiotic local treatment of the vaginal mucosa, Str. uberis, C. albicans, P. aeruginosa, S. aureus, P. mirabilis and E. cloacae were completely eliminated these microorganisms were not detected in the swabs after the end of the experiment (Figure 3).

\section{Dynamics of the number of animals with S. aureus, C. albicans and} E. coli against the background of the use of phytobiotic

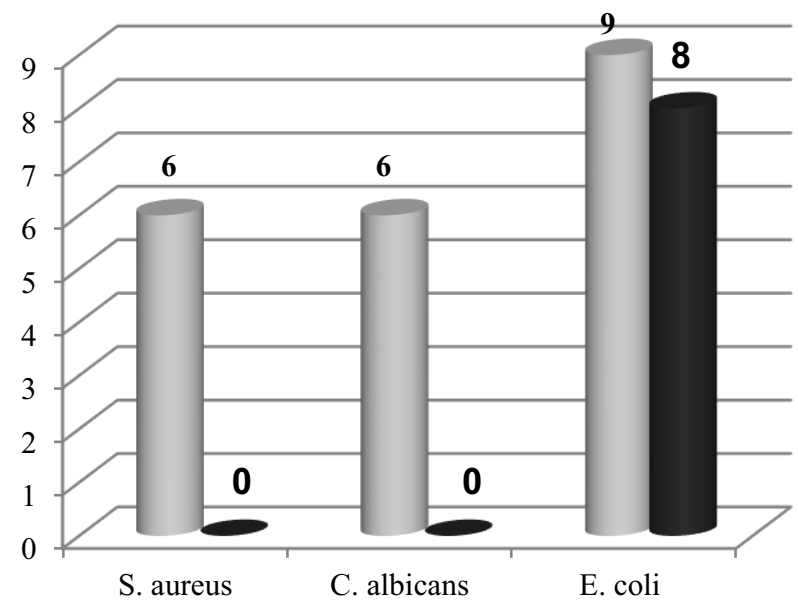

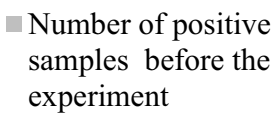

- Number of positive samples after the experiment

Fig.3. Dynamics of the number of animals with S. aureus, C. albicans and E. coli against the background of the phytobiotic use.

The number of heads with positive tests for E. coli decreased slightly. The use of the phytobiotic did not affect P. vulgaris and Bacillus spp., they were detected in the swabs of 4 cows both before and after the end of the experiment. There was also a noticeable 
improvement in the condition of the vaginal mucosa of cows after the phytobiotic use. Before the start of the experiment, 9 out of 10 animals had signs of mucosal inflammation, after the experiment, such signs were detected only in 2 animals, while their intensity was less pronounced, there was no hyperemia, and the secretion became more scarce. This fact is presumably related to the ability of secondary $\mathrm{N}$. sativa metabolites to inhibit the lipoxygenase and cyclooxygenase pathways in arachidonic acid metabolism, as well as to the immunomodulatory effect of the metabolites mediated through the enhancement of the function of the immunity cellular link (T- and NK cells) [12, 13]. A decrease in the induction of tissue mediators of inflammation in combination with the modulation of the cellular immune response, as well as the elimination of a number of facultative pathogens, had a noticeable sanitizing effect on the vaginal mucosa in cows, which was manifested by the disappearance of clinical signs of inflammation already on the 5th-6th day of the phytobiotic use.

\section{Conclusions}

The results of the studies showed that the phytobiotic based on essential oil substances Nigella sativa L. had inhibitory activity against both multi-antibiotic-resistant cultures of P. aeruginosa and $\mathrm{S}$. aureus in vitro, and against these microorganisms in the microbiocenoses of the vaginal mucosa of productive cows. In addition, the antibacterial effect of the phytobiotic on such pathogens as Str. uberis, E. cloacae, C. albicans, P. mirabilis was established - these microorganisms were completely eliminated from the cow mucosa after topical application of the preparation. Thus, the phytobiotic for external use based on active metabolites of Nigella sativa L. can be considered promising for use as the main or auxiliary agent for the treatment of dysbiosis of the vaginal mucosa in cows in the postpartum period.

\section{Acknowledgments}

The research is executed at the expense of a grant of the Russian scientific fund (project No. 18-16-00040).

\section{References}

1. K.N. Galvão, R.C. Bicalho, S.J. Jeon, Dairy Sci. 102(12), 11786-11797 (2019) doi: 10.3168/jds.2019-17106. Epub 2019 Oct 3.

2. I.M. Donnik, I.A. Shkuratova, Herald of the Russian Academy of Sciences. 87(2), 139142 (2017)

3. E.A. Solenova A. Flavonoids, Acta Medica Eurasica 3, 50-57 (2017)

4. O.A. Grebennikova, A. E. Paly, L. A. Khlypenko, V. D. Rabotyagov, Journal "Orbital" 1, 21-28 (2017)

5. A.A. Mostafa, A.B. Mostafa Abdulaziz et al., Saudi Journal of Biological Sciences. 25(2), 361-366 (2017)

6. A.G. Parreira, E.J.C. Azevedo, F.R. Pereira et al., Arch Biomed Eng \& Biotechnol. 1(5), 1-7 (2019) ABEB.MS.ID.000524

7. V.S. Grinev, A.A. Shirokov, N.A. Navolokin et al., Russian Journal of Bioorganic Chemistry 42(7), 770 -776 (2016) 
8. M.F. Ahmad, F.A. Ahmad, S.A. Ashraf, H.H. Saad, S. Wahab, M.I. Khan, M. Ali, S Mohan, K.R. Hakeem, M.T. Athar, Journal of Herbal Medicine (2020), doi: https://doi.org/10.1016/j.hermed.2020.10040

9. D. Alsaidy, IJARES 2(1), 1081-1086 (2014)

10. Clinical recommendations. Determination of the susceptibility of microorganisms to antimicrobials (Clinical guidelines. Determination of the sensitivity of microorganisms to antimicrobial drugs), (2015) URL:https://www.antibiotic.ru/files/321/clrecdsma2018.pdf (date of access 02.09.2020)

11. M.L. Salem, International immunopharmacology 5(13-14), 1749-1770 (2005)

12. H.N. Pise, S.L. Padwal, National Journal of Physiology, Pharmacy and Pharmacology 7(7), 707 (2017)

13. B.G. Andriukov, T.S. Zaporozhets, N.N. Besednova, Antibiotics and chemotherapy 12, 44-55 (2018)

14. S. Darakhshan, A.B. Pour, A.H. Colagar, S. Sisakhtnezhad, Pharmacol Res 95, 138$158(2015)$ 